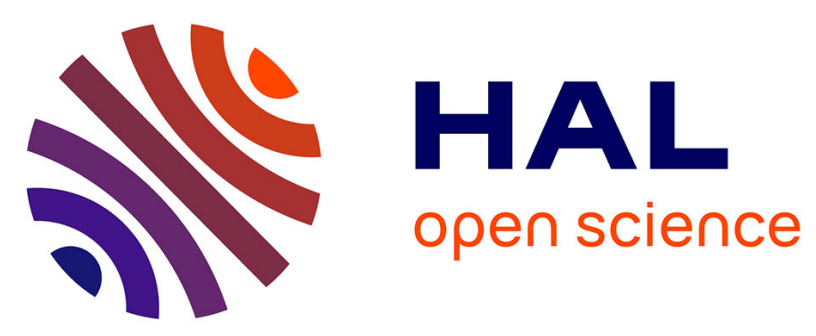

\title{
Dimension reduction-based attributes selection in no-reference learning-based image quality algorithms
}

Christophe Charrier, Abdelhakim Saadane, Christine Fernandez-Maloigne

\section{To cite this version:}

Christophe Charrier, Abdelhakim Saadane, Christine Fernandez-Maloigne. Dimension reductionbased attributes selection in no-reference learning-based image quality algorithms. Image Quality and System Performance Conference, IS\&T Electronic Imaging, Jan 2017, Burlingame, United States. pp.15 - 20, 10.2352/ISSN.2470-1173.2017.12.IQSP-219 . hal-01595888

\section{HAL Id: hal-01595888 \\ https://hal.science/hal-01595888}

Submitted on 27 Sep 2017

HAL is a multi-disciplinary open access archive for the deposit and dissemination of scientific research documents, whether they are published or not. The documents may come from teaching and research institutions in France or abroad, or from public or private research centers.
L'archive ouverte pluridisciplinaire HAL, est destinée au dépôt et à la diffusion de documents scientifiques de niveau recherche, publiés ou non, émanant des établissements d'enseignement et de recherche français ou étrangers, des laboratoires publics ou privés. 


\title{
Dimension reduction-based attributes selection in no-reference learning-based image quality algorithms
}

\author{
Christophe Charrier ${ }^{1}$, Abdelhakim Saadane ${ }^{2}$, Christine Fernandez-Maloigne ${ }^{3}$ \\ ${ }^{1}$ Normandie Univ, UNICAEN, ENSICAEN, CNRS, GREYC, 14000 Caen, FRANCE \\ 2 Université de Nantes, XLIM UMR CNRS 7252 \\ ${ }^{3}$ Université de Poitiers, XLIM UMR CNRS 7252
}

\begin{abstract}
No-reference image quality metrics are of fundamental interest as they can be embedded in practical applications. The main goal of this paper is to define a new selection process of attributes in no-reference learning-based image quality algorithms. To perform this selection, attributes of seven well known no-reference image quality algorithms are analyzed and compared with respect to degradations present into the image. To assess the performance of these algorithms, the Spearman Rank Ordered Correlation Coefficient (SROCC) is computed between the predicted values and the MOS of three public databases. In addition, an hypothesis test is conducted to evaluate the statistical significance of performance of each tested algorithm.
\end{abstract}

\section{Introduction}

Lossy image compression techniques such as JPEG2000 allow high compression rates, but only at the cost of some perceived degradation in image quality. The way to evaluate the performance of any compression scheme is a crucial step, and more precisely available ways to measure the quality of compressed images. There is a very rich literature on image quality criteria, generally dedicated to specific applications (optics, detector, compression, restoration, ... . ). From several years, a number of researches have been conducted to design robust No-Reference Image Quality Assessment (NR-IQA) algorithms, claiming to have made headway in their respective domains. NR-IQA algorithms generally follow one of three trends. 1) Distortion-specific approaches: These employ a specific distortion model to drive an objective algorithm to predict a subjective quality score. These algorithms quantify one or more distortions such as blockiness $[1,2]$, blur [3, 4] or ringing [5, 6] and score the image accordingly. 2) Training-based approaches: these train a model to predict the image quality score based on a number of features extracted from the image $[7,8,9,10,11]$. 3) Natural scene statistics (NSS) approaches: these rely on the hypothesis that images of the natural world (i.e. distortion free images) occupy a small subspace of the space of all possible images and seek to find a distance between the test image and the subspace of natural images [11, 12, 13].

Yet, all existing algorithms fail for at least one to correctly predict the quality of an image when degradation has not been learned. Degradation is not necessarily the same for the trial algorithms. Taking into account those failure cases will help to design a robust NR-image quality assessment algorithm.

In this paper, we develop a NR-image quality assessment algorithm based on an attribute selection approach. All the attributes are extracted from usually used and well-known NR-IQA training-based algorithms. The trial NR-IQA algorithms used are the following: 1) BIQ-An [12], 2) BIQI [11], 3) DIIVINE [14], 4) BLIINDS [15], 5) BLIINDS-2 [16], 6) BRISQUE [17], 7) NIQE [18]. The implementations of the algorithms were either publicly available on the Internet or obtained from the authors. A features vector is constructed from all attributes and a attribute selection process is designed to only keep descriptors that have been identified as meaningful with respect to the image. The rest of paper is organized as follows. Section II presents the used databases. The proposed new NR-IQA is given in Section III. The performance is evaluated in Section IV and a conclusion is made in Section V.

\section{NR-IQA algorithms and image databases Trial NR-IQA schemes}

All attributes considered in this paper are extracted from NRIQA learning-based metrics summarized in Table 1. This table also gives the number of attributes used by each algorithm.

\begin{tabular}{|l|l|l|}
\hline \hline $\begin{array}{l}\text { NR-IQA algo- } \\
\text { rithm }\end{array}$ & $\begin{array}{l}\text { comments at- } \\
\text { tributes }\end{array}$ \\
\hline \hline BIQI & $\begin{array}{l}\text { Machine learning-based ap- } \\
\text { proach (SVM). }\end{array}$ & 18 \\
\hline DIIVINE & $\begin{array}{l}\text { Classification (SVM) and Re- } \\
\text { gression (SVR) }\end{array}$ & 88 \\
\hline BLIINDS & $\begin{array}{l}\text { Machine learning-based ap- } \\
\text { proach (Probabilistic model) }\end{array}$ & 12 \\
\hline BLIINDS-II & $\begin{array}{l}\text { Machine learning-based ap- } \\
\text { proach (Probabilistic model) }\end{array}$ & 12 \\
\hline BIQ-An & $\begin{array}{l}\text { Renyi entropy measure along } \\
\text { various orientations }\end{array}$ & 6 \\
\hline NIQE & $\begin{array}{l}\text { Space domain natural scene } \\
\text { statistic model }\end{array}$ & 18 \\
\hline BRISQUE & $\begin{array}{l}\text { Natural scene statistic-based } \\
\text { distortion-generic }\end{array}$ & 18 \\
\hline
\end{tabular}

Table 1: NR-IQA trial Algorithms

\section{Databases description}

To provide comparison of NR-IQA algorithms, three publicly available databases are used: 1) LIVE database [19], 2) TID2008 database [20] and 3) CSIQ image database [21]. The LIVE database contains 29 original images on which five kinds of distortions have been applied to generate 770 degraded images. The TID2008 database contains 1700 distorted versions of 25 original images (from Kodak Lossless True Color Image Suite) applying 17 distortion types. The CSIQ database consists of 30 


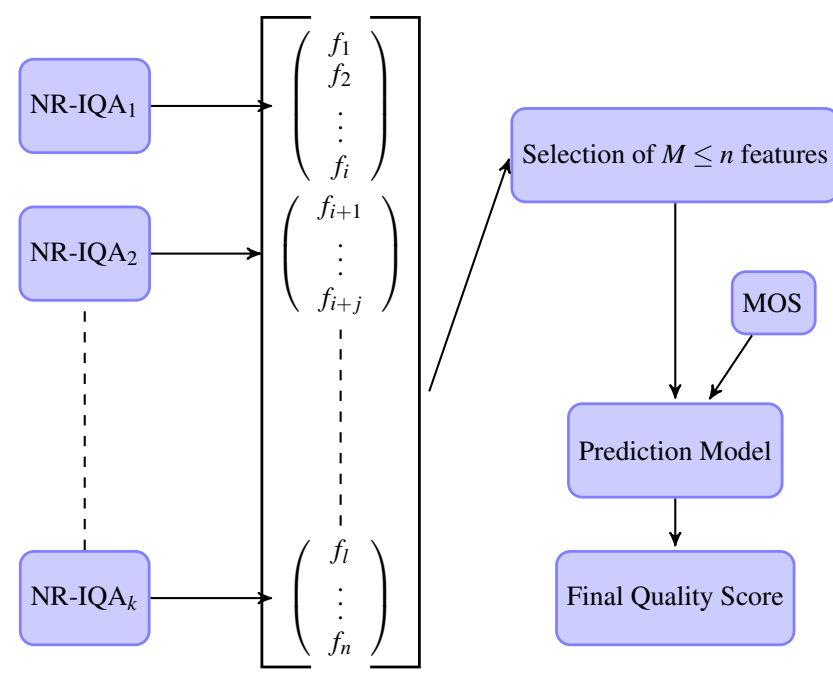

new vector of $n$ extracted features from each NRIQA method

Figure 1. General scheme of the proposed NR-IQA algorithm based on combination of features.

original images, each distorted using six types of distortions at four to five different levels as detailed above.

\section{Tampere Image Database 2008 (TID2008)}

The Tampere Image Database 2008 (TID2008) was created in 2008, for the evaluation of full-reference image quality assessment metrics and developed at the Tampere University of Technology, in Finland. The TID2008 contains 25 reference images and 1700 distorted images corresponding at 17 types of distortions on 4 levels. Reference images are obtained by cropping from Kodak Lossless True Color Image Suite (http://r0k.us/graphics/kodak/). All images are in BMP format at $384 X 512$ pixels resolution. A software which developed based on Swiss System is using to conduct the psychophysical experiment. Mean Opinion Scores (MOS) were calculated from the subjective data. The MOS were obtained from the results of 838 experiments carried out by observers from three countries: Finland, Italy, and Ukraine (251 experiments have been carried out in Finland, 150 in Italy, and 437 in Ukraine). Totally, the 838 observers have performed 256428 comparisons of visual quality of distorted images or 512856 evaluations of relative visual quality in image pairs. Subjective evaluations were made in standard PC laboratory or via the internet. http://www. computervisiononline.com/ dataset/tid2008-tampere-image-database-2008

\section{Categorical Subjective Image Quality (CSIQ) Database}

The Categorical Subjective Image Quality (CSIQ) Database was released by the Computational Perception and Image Quality Lab, at the Oklahoma state University, in 2010. It consists of 30 original images, each is distorted using six different types of distortions at four to five different levels of distortion. CSIQ images are subjectively rated base on a linear displacement of the images across four calibrated LCD monitors placed side by side with equal viewing distance to the observer.
The database contains 5000 subjective ratings from 35 different observers, and ratings are reported in the form of Difference of Mean Opinion Scores (DMOS). Subjective evaluations were made on pre-calibrated display and viewing distance is $70 \mathrm{~cm}$. http://vision.okstate.edu/?loc=vqa\_samviq

\section{NR-IQA scheme design General Scheme}

All the trial NR-IQA methods applies the same principle: after computing a set of different features, a combination of this set is performed in different ways. In this paper, the design a NRIQA algorithm is based on a new selection process of relevant attributes provided by different common NR-IQA methods. Fig 1 displays the general scheme of the proposed method. From all trial NR-IQA schemes, all features are computed. This yields to a set of $n$ features. From this initial set a subset of $M$ attributes is generated (under the constraint that $M \leq n$ ) in order to keep the most relevant features with respect to some criteria. Then a combination of these $M$ features is performed to design a NRIQA algorithm scoring with a high correlation level with human judgments of image quality.

\section{Attributes selection criteria}

Given a set of visual attributes describing an image, we use a Manifold Learning method to project the data on a new lowdimensional space. Thus, nonlinear new discriminant features of the input data are yielded. The obtained low dimensional submanifold is used as a new representation that is transmitted to design the proposed NR-IQA algorithm.

When data objects are described by a large number of features (i.e., the data are of high dimension) it is often beneficial to reduce the dimension of the data. Dimension reduction can be beneficial not only for reasons of computational efficiency but also because it can improve the accuracy of the analysis. To deal with this issue, dimension reduction techniques are often applied as a data pre-processing step or as part of the data analysis to simplify the data model. This typically involves the identification of a suitable low-dimensional representation for the original high-dimensional data set. Dimensionality reduction methods can be divided into two sets whether the transformation is linear or nonlinear. We detail here the principles of Laplacian Eigenmaps (LE)[22] that will be used in this study.

Let $X=\left\{\mathbf{x}_{1}, \mathbf{x}_{2}, \cdots, \mathbf{x}_{n}\right\} \in \mathbb{R}^{p}$ be $n$ sample vectors. Dimensionality reduction consists in finding a new low-dimensional representation in $\mathbb{R}^{p}$ with $q \ll p$.

Given a neighborhood graph $G$ associated to the vectors of $X$, one considers its adjacency matrix $W$ where weights $W_{i j}$ are given by a Gaussian kernel

$$
W_{i j}=k\left(\mathbf{x}_{i}, \mathbf{x}_{j}\right)=e\left(-\frac{\left\|\mathbf{x}_{i}-\mathbf{x}_{j}\right\|^{2}}{\sigma^{2}}\right) .
$$

Let $D$ denote the diagonal matrix with elements $D_{i i}=\sum_{j} W_{i j}$ and $\Delta$ denote the un-normalized Laplacian defined by $\Delta=D-W$.

Laplacian Eigenmaps dimensionality reduction consists in searching for a new representation $\left\{\mathbf{y}_{1}, \mathbf{y}_{2}, \cdots, \mathbf{y}_{n}\right\}$ with $\mathbf{y}_{i} \in \mathbb{R}^{n}$, obtained by minimizing $\frac{1}{2} \sum_{i j}\left\|\mathbf{y}_{i}-\mathbf{y}_{j}\right\|_{2} W_{i j}=$ $\operatorname{Tr}\left(\mathbf{Y}^{T} \Delta \mathbf{Y}\right)$ with $\mathbf{Y}=\left[\mathbf{y}_{1}, \mathbf{y}_{2}, \cdots, \mathbf{y}_{n}\right]$. This cost function encourages nearby sample vectors to be mapped to nearby outputs. 


\begin{tabular}{|c||c|c|c|c|c|c|c|c|}
\hline \hline CSIQ subset & BIQI & DIIVINE & BLIINDS & BLIINDS-II & BIQ-An & NIQE & BRISQUE & FSIQI \\
\hline JP2k & 0.708 & 0.830 & 0.575 & 0.895 & 0.460 & 0.906 & 0.866 & 0.901 \\
JPEG & 0.867 & 0.799 & 0.264 & 0.901 & 0.012 & 0.883 & 0.903 & 0.902 \\
Gaussian Noise & 0.324 & 0.176 & 0.293 & 0.379 & 0.091 & 0.299 & 0.252 & 0.402 \\
Add. Gaussian Pink Noise & 0.879 & 0.866 & 0.555 & 0.801 & 0.303 & 0.810 & 0.925 & 0.917 \\
Gaussian Blur & 0.771 & 0.871 & 0.774 & 0.891 & 0.739 & 0.892 & 0.903 & 0.894 \\
Global Contrast Decrement & 0.585 & 0.396 & 0.078 & 0.012 & 0.767 & 0.232 & 0.029 & 0.156 \\
\hline Cumulative subsets & 0.619 & 0.596 & 0.170 & 0.577 & 0.286 & 0.628 & 0.566 & 0.699 \\
\hline \hline
\end{tabular}

Table 2: SROCC values computed between predicted scores and MOS values for the CSIQ Images database.

\begin{tabular}{|l||c|c|c|c|c|c|c|c|}
\hline \hline TID2008 subset & BIQI & DIIVINE & BLIINDS & BLIINDS-II & BIQ-An & NIQE & BRISQUE & FSIQI \\
\hline $\begin{array}{l}\text { Additive noise in color compo- } \\
\text { nents is more intensive than addi- } \\
\text { tive noise in the luminance com- } \\
\text { ponent }\end{array}$ & 0.46 & 0.366 & 0.441 & 0.778 & 0.117 & 0.742 & 0.495 & 0.781 \\
\hline Spatially correlated noise & 0.589 & 0.722 & 0.245 & 0.446 & 0.311 & 0.758 & 0.584 & 0.746 \\
\hline Masked noise & 0.897 & 0.872 & 0.688 & 0.866 & 0.175 & 0.854 & 0.623 & 0.895 \\
\hline High frequency noise & 0.787 & 0.864 & 0.583 & 0.586 & 0.016 & 0.687 & 0.582 & 0.855 \\
\hline Impulse noise & 0.406 & 0.188 & 0.039 & 0.719 & 0.110 & 0.812 & 0.723 & 0.788 \\
\hline Quantization noise & 0.747 & 0.824 & 0.663 & 0.839 & 0.570 & 0.817 & 0.799 & 0.772 \\
\hline Image denoising & 0.306 & 0.759 & 0.509 & 0.760 & 0.481 & 0.605 & 0.570 & 0.796 \\
\hline JPEG2000 transmission errors & 0.367 & 0.021 & 0.211 & 0.606 & 0.262 & 0.493 & 0.260 & 0.489 \\
\hline Non eccentricity pattern noise & 0.010 & 0.032 & 0.033 & 0.142 & 0.067 & 0.016 & 0.163 & 0.131 \\
\hline $\begin{array}{l}\text { Local blockwise distortions of } \\
\text { different intensity }\end{array}$ & 0.022 & 0.060 & 0.150 & 0.457 & 0.124 & 0.183 & 0.175 & 0.421 \\
\hline Mean shift (intensity shift) & 0.024 & 0.050 & 0.355 & 0.057 & 0.384 & 0.138 & 0.091 & 0.312 \\
\hline \hline Cumulative subsets & 0.282 & 0.145 & 0.072 & 0.342 & 0.037 & 0.134 & 0.225 & 0.451 \\
\hline
\end{tabular}

Table 3: SROCC values computed between predicted scores and MOS values for the TID Images database subsets for which associated degradations are not common with LIVE database subsets.

This is achieved by finding the eigenvectors $\mathbf{y}_{1}, \mathbf{y}_{2}, \cdots, \mathbf{y}_{n}$ of matrix $\Delta$. Dimensionality reduction is obtained by considering the $q$ lowest eigenvectors (the first eigenvector being discarded) with $q \ll p$ and is defined by the following operator $h_{L E}: \mathbf{x}_{i} \rightarrow\left(y_{2}(i), \cdots, y_{q}(i)\right)$ where $y_{k}(i)$ is the $i^{\text {th }}$ coordinate of eigenvector $\mathbf{y}_{k}$.

\section{Final quality score calculation}

Once the laplacian eigenmaps is performed on initial data, all generated eigenvectors are descending ordered by their associated eigenvalues. Then data are projected on this eignespace. Let $\tilde{f}_{i}$ ) be the i-th projected data. The final quality score based on a selection process, namely Feature Selection-based Image Quality Index (FSIQI) can be expressed as a $M$-parameters function :

$$
\mathrm{FSIQI}=\prod_{i=1}^{M}\left(\tilde{f}_{i}\right)^{\alpha_{i}}
$$

where $\alpha_{i}$ is a weight factor applied to the $\mathrm{i}$-th projected data $\tilde{f}_{i}$ ) and $M$ is the number of considered attributes. The main objective is to find optimal exponent values of (2).

\section{The associated error function}

From (2), the search for the exponent values seeks to minimize the error function

$$
\begin{aligned}
& E\left(\alpha_{i} ; i=1, \cdots, M\right)= \\
& \quad \min \left(\sum_{j=1}^{K}\left(\mathrm{DMOS}_{j}-\mathrm{FSIQI}_{j}\left(\alpha_{i}\right)\right)^{2}\right)
\end{aligned}
$$

where $K$ is the number of tested images for which the DMOS values are provided, and $\mathrm{FSIQI}_{j}($.$) are the quality computed rates$ obtained following a logistic regression as depicted in [23].

In other words, the goal is to estimate the $M$ exponent values that minimize the error function $E($.$) . Since the error function is$ non-convex and may contain numerous local optima, the choice of search strategy to optimize it is important.

\section{Search strategy}

The Genetic Algorithm (GA) is a population-based stochastic search procedure that finds exact or approximate solutions to optimization and search problems. Modeled on the mechanisms of evolution and natural genetics, genetic algorithms uses directed random searches to locate optimal solutions in multimodal landscapes. Their basic principles were first introduced by Holland in 1975 [24].

Usually, a simple GA is composed of three operations: selection, genetic operation, and replacement. GAs use a population, which is composed of a group of chromosomes, to represent the solutions of the system. Defining the solution representation of the system is the first task when applying GAs. The solution in the problem domain can then be encoded into the chromosome in the GA domain, and vice versa. Initially, a population is randomly generated. The fitting function then uses values from objective functions to evaluate the quality of fit of each chromosome. Next, a particular group of chromosomes is chosen from the population to be parents. The offsprings are then generated from these parents using genetic operations (crossover and mutation). The 


\begin{tabular}{|c||c|c|c|c|}
\hline \hline & BIQI & DIIVINE & BLIINDS & BLIINDS-II \\
\hline FSIQI & $11-11-111111$ & $1---10-11111$ & $111111111-1$ & $-1-1-0-0--11$ \\
\hline \hline & BIQ-An & NIQE & BRISQUE & FSIQI \\
\hline FSIQI & 11111111101 & $---1001-1111$ & $1111--110111$ & ---------- \\
\hline
\end{tabular}

Table 4: Statistical significance matrix of NR-IQA/DMOS on TID database subsets. Each entry in the table is a codeword consisting of 12 symbols. The position of the symbol represents the tested subsets as mentioned in the first column of Table 3 . Each symbol gives the result of the hypothesis test on the subset: ' 1 ' means that the algorithm for the row is statistically better that the algorithm for the column, ' $O$ ' means it is worse, and '-' means it is indistinguishable.

\begin{tabular}{|c||c|c|c|c|c|c|c|c|}
\hline \hline & BIQI & DIIVINE & BLIINDS & BLIINDS-II & BIQ-An & NIQE & BRISQUE & FSIQI \\
\hline BIQI & ----- & $--0-01-$ & $11---1$ & $100-01-$ & $111-1-1$ & $100-01-$ & $000-010$ & 0000010 \\
DIIVINE & $--1-10-$ & ------ & $111--01$ & $100--0-$ & $111-101$ & $10--00-$ & $00--000$ & $0000-10$ \\
BLIINDS & $00---00$ & $000--10$ & ----- & $000---0$ & $1-1-10-$ & $000-0-0$ & $000-0-0$ & $00000-1$ \\
BLIINDS 2 & $011-10-$ & $011--1-$ & $111---1$ & ----- & $111-101$ & $----0--$ & $0---0-0$ & $0--0-00$ \\
BIQ-An & $000-0-0$ & $000-010$ & $0-0-01-$ & $000-010$ & ------ & $000-010$ & $000-010$ & 0000010 \\
NIQE & $011-10-$ & $01--11-$ & $111-1-1$ & $----1--$ & $111-101$ & ------ & $0----0$ & $--00-1-$ \\
BRISQUE & $111-101$ & $11--111$ & $111-1-1$ & $1---1-1$ & $111-101$ & $1----1$ & ------ & $--0--00$ \\
FSIQI & 1111101 & $1111-01$ & $11111-0$ & $1--1-11$ & 1111101 & $--11-0-$ & $--1--11$ & ------ \\
\hline
\end{tabular}

Table 5: Statistical significance matrix of NR-IQA/DMOS on CSIQ database subsets. Each entry in the table is a codeword consisting of 7 symbols. The position of the symbol represents the tested subsets: Additive pink Gaussian noise, JP2K, JPEG, Gaussian noise, Gaussian blur, Global Contrast Decrements, all data. Each symbol gives the result of the hypothesis test on the subset: ' 1 ' means that the algorithm for the row is statistically better that the algorithm for the column, ' 0 ' means it is worse, and '-' means it is indistinguishable.

fitness of the offspring is then evaluated and used in replacement processes in order to replace the chromosomes in the current population by the selected off-spring. The GA cycle is then repeated until a desired termination criterion is satisfied, or the objective value is below the threshold.

In this paper, $M$ is the number of features used to compute FSIQI values. In that case, the GA domain represents a $M$-dimensional space in which one point is expressed as $\left(\alpha_{i}, \cdots, \alpha_{M}\right)$, and the fitness function is defined by (3).

\section{Performance Evaluation Apparatus}

FSIQI results are compared to results provided by all trial NR-IQA algorithms. To preform this evaluation, the Spearman Rank Order Correlation Coefficient (SROCC) is computed between the DMOS values and the predicted scores obtained from NR-IQA algorithms. Since the LIVE database has been used to find optimal $\alpha_{i}$ and $M$ parameters, both TID2008 and CSIQ databases will serve as test set.

In addition, to ascertain which differences between NR-IQA schemes performance are statistically significant, we applied an hypothesis test using the residuals between the DMOS values and the ratings provided by the IQA algorithms. This test is based on the t-test that determines whether two population means are equal or not. This test yields us to take a statistically-based conclusion of superiority (or not) of an NR-IQA algorithm.

\section{How to choose $M$ value ?}

In order to investigate the optimal $\alpha_{i}$ values and the $M$ parameters needed to design FSIQI algorithm, we used the LIVE database as training set to compute initial features.

Finding the number $M$ is rely to figure out the dimension $i$ of the eigenspace. This can be expressed as $\forall i \in[1, \ldots, n]$,

$$
\arg \min _{i}(\operatorname{SROCC}(\operatorname{FSIQI}(i))-\operatorname{SROCC}(\operatorname{FSIQI}(i+1))<\varepsilon)
$$

In this study $\varepsilon=0.1$, and we found that for eigenspaces of more than 8 dimensions, corresponding SROCC values are very close and consecutive differences are lower than $\varepsilon$. This, only the 8 first eigenvectors are considered to define the eigenspace on which initial features will be projected on.

\section{Comparison results}

In order to evaluate the performance of the FSIQI algorithm, a comparison of SROCC values obtained from all trial NR-IQA schemes is performed on both TID2008 and CSIQ database. Table 2 presents obtained results when SROCC is computed between predicted scores and MOS values for the CSIQ Images database for all trial NR-IQA algorithms and the proposed one. One can observe that the performance of the trial NR-IQA algorithm vary according to degradation even if NIQUE and BRISQUE seem to be better than the remaining ones. However, FSIQI the proposed method, presents high performance whatever the degradation (except for the last one (Global contrast decrement)).

Table 3 presents obtained results when SROCC is computed between predicted scores and MOS values for the TID Images database subsets which are not common to the LIVE database and for all trial NR-IQA algorithms and the proposed one. One can also notice that the performance of the 7 trial NR-IQA algorithms are highly dependent on the considered degradation. BIQI performs better for masked noise, DIIVINE for high frequency noise, BLIINDS-II for additive noise, quantization noise and image denoising and JPEG2000 transmission errors, NIQE for spatially correlated noise and impulse noise. By contrast, FSIQI, equals or outperforms the best trial NR-IQA algorithm regardless of the considered degradation. Moreover, the SROCC value of FSIQI metric is equal or greater than 0.772 for the first seven degradations. For a learning-based metric as FSIQI, this performance remains very interesting since the training set and the test set have no common degradations.

Table 4 gives obtained results when a One-sided t-test is 


\begin{tabular}{|c||c|c|c|c|c|c|c|c|}
\hline \hline Algorihm & BIQI & DIIVINE & BLIINDS & BLIINDS-II & BIQ-An & NIQE & BRISQUE & FSIQI \\
\hline time & 6.95 & 38.39 & 509.82 & 131.25 & 1.97 & 0.83 & 0.75 & 376.54 \\
\hline
\end{tabular}

Table 6: Comparison of computational time (in second/image)

used to provide statistical significance of NR-IQA/DMOS on TID database. Each entry in this table is coded using 12 symbols. The position of each symbol corresponds to one subset of the TID database as expressed in table 3. Each symbol gives the result of the hypothesis test on the subset. If the symbol equals ' 1 ', the NR-IQA on the row is statistically better than the NR-IQA on the column (' 0 ' means worse, '-' is used when NR-IQAs are indistinguishables). On can observe that difference between quality scores predicted with FSIQI and any trail algorithms is most of the time significant when the entire database is considered or when any subset is used. Since the learning process is applied on LIVE database to design FSIQI, this result illustrates its capability to judge well image quality when applied degradations are not learnt.

Tables 5 shows similar results when CSIQ database is considered. The position of the symbol represents the tested subsets: Additive pink Gaussian noise, JP2K, JPEG, Gaussian noise, Gaussian blur, Global Contrast Decrements, all data. Considering the global performance on all data subsets, FSIQI over performs all other tested NR-IQA methods, except BLIINDS. Yet for one particular degradation (Global Contrast Decrements associated to the 6th digit), FSIQI seems to be less efficient than other trial algorithms to predict quality scores.

Finally, to compare the computational complexity of the proposed algorithm, we measured the average computation time required to assess an image of size $512 \times 578$ (using a computer with Intel Core-I7 processor at $2.2 \mathrm{GHz}$ ). Table 6 reports the measurement results, which are rough estimates only, as no code optimization has been done on our Matlab implementations. It can be observed that the proposed method is one of the time consuming method but less than BLIINDS. This is not really surprising, since FSIQI consists in using all attributes of prior NR-IQA algorithms (around 180 attributes) to compute final score in another space.

Yet, one can remark that since the dimensionality of the eigenspace is to be equal 8 , this means that the fundamental NRIQA problem exists in a space of around 8 attributes.

\section{Conclusion}

We have used a selection process of quality attributes based on manifold to design a new NR-IQA scheme, named FSIQI. Considering all attributes used by seven trial NR-IQA algorithms, a high dimensional feature vector is obtained and the reduced applying laplacian eigenmaps. We have shown the new FSIQI algorithm only needs eight features to performs well in this new representation space of features. The method correlates highly with human perception of quality, and outperforms recent NR-IQA algorithms.

\section{References}

[1] R. Muijs and I. Kirenko, "A no-reference blocking artifact measure for adaptive video processing," in European Signal Processing Conference (Eusipco), 2005.

[2] X. Song and Y. Yang, "A new no-reference assessmet metric of blocking artefacts on HVS masking effect," in Interna- tional Congress on Image ans Signal Processing, pp. 1-6, 2009.

[3] Z. ParvezSazzad, Y. Kawayoke, and Y. Horita, "Noreference image quality assessment for jpeg2000 based on spatial features," Signal Processing: Image Communication 23(4)(4), pp. 257-268, 2008.

[4] R. Barland and A. Saadane, "Blind quality metric using a perceptual map for jpeg2000 compressed images," in International Conference on Image Processing (ICIP), 2006.

[5] L. Hantao, N. Klomp, and I. Heynderickx, "A no-reference metric for perceived ringing artifacts in images," IEEE Transactions on Circuits and Systems for Video Technology 20(4), pp. 529-539, 2010.

[6] S. Hu, Z. Pizlo, and J. P. Allebach, "Jpeg ringing artifact visibility evaluation," in Proc. SPIE 9016, Image Quality and System Perfromance XI, 2014.

[7] H. Luo, "A training-based no-reference image quality assessment algorithm," in International Conference on Image Processing (ICIP), pp. 2973-2976, 2004.

[8] Y. R. Tsoy, V. G. Spitsyn, and A. V. Chernyavsky, "Noreference image quality assessment through interactive neuroevolution," in International Conference on Computer Graphics and Vision, pp. 23-27, 2007.

[9] R. V. Babu, S. Suresh, and A. Perkis, "No-reference JPEG image quality assessment using GAP-RBF," Signal Processing 87 (6)(6), pp. 1493-1503, 2007.

[10] A. K. Moorthy and A. C. Bovik, "Visual importance pooling for image quality assessment," IEEE J. Selected Topics in Signal Process., Special Issue on Visual Media Quality Assessment 3(2), pp. 193-201, 2009.

[11] A. K. Moorthy and A. C. Bovik, "A two-step framework for constructing blind image quality indice," IEEE Signal processing letters, 17(5), pp. 513-516, 2010.

[12] S. Gabarda and G. Cristobal, "Blind image quality assessment through anisotroy," JOSA 24(12), pp. B42-B51, 2007.

[13] T. Brandao and M. P. Queluz, "No-reference image quality assessment based on dct-domain statistics," Signal Processing 88(4), pp. 822-833, 2008.

[14] A. K. Moorthy and A. C. Bovik, "Blind image quality assessment: From natural scene statistics to perceptual quality," IEEE Transactions Image Processing 20(12), pp. 33503364, 2011.

[15] M. Saad, A. C. Bovik, and C. Charrier, "A DCT statisticsbased blind image quality index," IEEE Signal Processing Letters 17(2), pp. 583-586, 2010.

[16] M. Saad, A. C. Bovik, and C. Charrier, "Blind image quality assessment: A natural scene statistics approach in the dct domain," IEEE Transactions on Image Processing 21(8), pp. 3339-3352, 2012.

[17] A. Mittal, A. K. Moorthy, and A. C. Bovik, "No-reference image quality assessment in the spatial domain," IEEE Transactions on Image Processing 21(12), pp. 4695-4708, 2012.

[18] A. Mittal, R. Soundararajan, and A. C. Bovik, "Making a 
"completely blind" image quality analyzer," IEEE Signal Process. Lett. 20(3), pp. 209-212, 2013.

[19] Laboratory for Image \& Video Engineering, University of Texas (Austin), "LIVE Image Quality Assessment Database," http://live.ece.utexas.edu/research/Quality , 2002.

[20] N. Ponomarenko, M. Carli, V. Lukin, K. E. ans J. Astola, and F. Battisti, "Color image database for evaluation of image quality metrics," in International Workshop on Multimedia Signal Processing, pp. 403-408, (Australia), Oct. 2008.

[21] E. C. Larson and D. M. Chandler, "Most apparent distortion: full-reference image quality assessment and the role of strategy," Journal of Electronic Imaging 19(1), pp. 011006-1 011006-21, 2010.

[22] M. Belkin and P. Niyogi, "Laplacien eigenmaps for dimensionality reduction and data representation," 15(6), pp. 1373-1396, 2003.

[23] VQEG, "Final report from the video quality experts group on the validation of objective models of video quality assessment," tech. rep., 2000.

[24] J. H. Holland, Adaptation in natural and artificial systems, MIT Press, Cambridge, MA, USA, 1992.

\section{Author Biography}

Christophe Charrier (M'10) received the M.S. degree from the Nantes University of Science and Technology, Nantes, France, in 1993, and the Ph.D. degree from the University Jean Monnet, Saint-Etienne, France, in 1998. He has been an Associate Professor with the GREYC laboratory at the University of Caen, France, since 2001. In 2008, he was a Visiting Scholar with the Laboratory for Image and Video Engineering, University of Texas, Austin. From 2009 to 2011, he was an Invited Professor with the Computer Department, University of Sherbrooke, Sherbrooke, $Q C$, Canada. He is now the head of the E-Payment \& Biometrics research team. His current research interests include digital image and video coding, processing, quality assessment, computational vision, and biometrics data quality.

Abdelhakim Saadane received his Ph.D. degree in Signal Processing and Telecommunications from University of Rennes1. He is currently associate professor at the Polytechnic school of Nantes University and a member of the SIC laboratory in XLIM Institute. His research activities are focused on the Human Visual System modeling and its use in applications such as coding, quality assessment, watermarking and user experience. He is a member of French National Color Imaging Group and serves as a member of division 8 of CIE.

Christine Fernandez-Maloigne is currently Vice-Rector of Poitiers University, in charge of International Relations. She is also director of a CNRS research federation (MIRES), which gathers 560 researchers in the South-West of France, in the area of mathematics, image processing, computer graphic, computer science and communication systems. Her research activities are focused on colour imaging, including fundamental researches about introduction of human visual system models in multiscale colour image processes as well as practical applications. She was cofounder member of the French National Colour Imaging Group (GFINC) in 2000, now part of the CFC (Centre Français de la Couleur). Currenlty, Christine Fernandez-Maloigne is French representative of the CIE Division 8 (Commission Internationale de l'Eclairage, Image Technology Division) since 2006 and secretary of this division since may 2015. She is also deputy Editorin-chief of JOSA A. 